\title{
Guerre et drogue au Liban. Entretien avec Antoine Boustany
}

Didier Bigo et Annie Laurent

\section{(2) OpenEdition}

12 Journals

Édition électronique

URL : http://journals.openedition.org/conflits/2015

DOI : 10.4000/conflits.2015

ISSN : $1777-5345$

Éditeur :

CCLS - Centre d'études sur les conflits lilberté et sécurité, L'Harmattan

Édition imprimée

Date de publication : 17 octobre 1991

ISSN : 1157-996X

Référence électronique

Didier Bigo et Annie Laurent, "Guerre et drogue au Liban. Entretien avec Antoine Boustany ", Cultures \& Conflits [En ligne], 03 | automne 1991, mis en ligne le 31 mars 2006, consulté le 30 mars 2021. URL: http://journals.openedition.org/conflits/2015; DOI : https://doi.org/10.4000/conflits.2015

Ce document a été généré automatiquement le 30 mars 2021.

Creative Commons License 


\title{
Guerre et drogue au Liban. Entretien avec Antoine Boustany
}

\author{
Didier Bigo et Annie Laurent
}

1 Antoine Boustany est Chef du département psychiatrie à la faculté de médecine de Beyrouth, et Médecin chef du centre médical spécialisé (toxicomanie-alcoolisme) à l'hôpital Saint Charles de Beyrouth.

2 C\&C : .A partir de quand est apparu l'usage de la drogue au Liban? Quelles furent les catégories sociales touchées et la guerre a-t-elle modifié la situation?

3 AB. : Oui, la guerre a modifié la situation et si l'on s'en tient à l'entendement étroit du terme "drogue", l'immense majorité des drogués se recrute parmi les combattants. En effet, c'est au sein des milices de tous bords, quelles que soient leur appartenance religieuse, politique, sociale et idéologique, que le phénomène s'est amplifié. Je ne dirais pas que c'est là qu'il a pris naissance, car quoique d'importance négligeable avant la guerre, les drogues avaient des adeptes et des consommateurs : très peu d'héroïnomanes, quelques cocaïnomanes recrutés dans le milieu intellectuel, artistique et parmi la bourgeoisie nantie, et un peu plus d'usagers de haschich parmi les autres catégories de population. Mais dès avant 1975 ce qui est à noter, c'est la disproportion qui existait au Liban entre une production importante et une consommation modérée. En revanche, si l'on confère un sens large, et d'ailleurs plus exact, au terme drogué, alors synonyme de dépendant, en y incluant les alcooliques, les pharmacomanes et surtout les "drogués de la violence" ou les "toxicomanes sans drogues" qui pullulent lors des périodes de guerre, notamment pendant les guerres civiles qui durent, les "drogués" se diversifient et se retrouvent dans toutes les couches et les catégories de la population.

4 Qui sont-ils ? Ce sont avant tout des citoyens soumis à l'horreur quotidienne dans toute sa laideur, au stress permanent, à l'angoisse continue, à la peur qui vous prend aux tripes sans relâche, à la culpabilité, à la brutalité étourdissante, à la cruauté impitoyable que l'on voit et que l'on vit, qui vous saisit, le tout étalé, non sur quelques semaines, ni sur quelques mois mais durant près de seize années. Ce sont donc des gens qui ont entendu, vu, subi et vécu d'une manière ou d'une autre ces situations de 
violence. Ce sont des personnes dont les mécanismes de défense ont été débordés et ont fléchi, et qui ont trouvé comme seul recours, l'usage de substances enivrantes, oublieuses ou perturbatrices de la réalité.

C\&C : Salim Nasr dans notre premier numéro a décrit les collusions entre les milices durant la guerre, mais peut-on être plus explicite sur les stratégies des dirigeants des milices à l'égard de la drogue?

6 A.B. : Les stratégies des dirigeants des milices à l'égard de la drogue ont évolué tout au long de la guerre. A mon avis, ceux qui ont été à la base de l'extension du phénomène parmi les combattants sont d'abord les anciens petits trafiquants, les dealers et les repris de justice. Ce sont eux les premiers qui ont frayé le chemin de la toxicomanie de masse surtout au moment où la situation se prêtait largement aux activités de prosélytisme. Leur cible favorite fut la catégorie des jeunes adolescents jetés au cœur de la bataille, et s'adonnant à ce jeu morbide de la guerre, à cette danse macabre, à cette aventure dont ils tiraient peur et jouissance. La drogue faisait alors partie de ce jeu (elle était sans doute une des règles), ajoutant ainsi une dimension de plaisir autodestructeur faisant régner une atmosphère malsaine et lugubre.

7 Mais ce qui a concouru à précipiter les choses, c'est le rôle alloué à ces trafiquants et autres psychopathes qui tournaient dans leur orbite, par les dirigeants des milices. La neutralisation politique de la troupe fit en sorte que les civils furent pris de cours devant les combats qui éclatèrent du jour au lendemain. Personne n'était mieux placé que ces repris de justice et psychopathes ayant l'expérience de la violence pour s'arroger un leadership au sein de ce rassemblement spontané de jeunes et moins jeunes qui manquaient totalement d'entraînement et d'expérience. Et, comme dans toute guerre civile, c'est la loi du plus fort qui prévalut sur celle du mérite et de l'intelligence. Ces petits leaders de quartier firent donc l'affaire des dirigeants des milices qui les embauchèrent comme "adjoints". Un accord implicite régnait alors, donnant les coudées franches aux trafiquants au sein des milices. Il est fort probable que la première année de la guerre et même l'année suivante, les dirigeants ne mesuraient pas vraiment la portée de cette attitude laxiste à l'égard du trafic et de la consommation de leurs subordonnés ainsi que le danger à plus long terme de cette politique de l'autruche, mais ensuite, prenant conscience progressivement de l'efficacité de la drogue comme arme de destruction du "facteur humain", les milices engagèrent une guerre que j'appellerai "Guerre des toxiques". La stratégie en était la suivante : il s'agissait d'intoxiquer les troupes de l'adversaire à petit feu, lentement mais sûrement et, dans ce but, il fut d'ailleurs demandé aux trafiquants de "frapper" leur commande de cocaïne avec de l'hérö̈ne. De la sorte, un adversaire "touché" était définitivement atteint par la dépendance. Il ne se contentait pas de traîner sa drogue derrière lui, il contribuait activement à la répandre autour de lui et devenait potentiellement un informateur pour son pourvoyeur. Bref, on faisait d'une pierre plusieurs coups!

8 Seulement là où le bât blesse et où les choses commencent à prendre une tournure de prime abord incompréhensible, c'est la passivité avec laquelle les dirigeants ont réagi à la propagation de l'usage de drogues de toutes sortes parmi leurs propres miliciens. Incompréhension de la stratégie adverse alors qu'ils l'utilisaient? Non. Ce n'est plus un secret pour personne que bon nombre de ces responsables, ou de ceux affublés de ce qualificatif, s'étaient en fait convertis ou plutôt avaient associé au(x) rôle(s) qu'ils prétendaient jouer, celui de trafiquant. Auraient-ils alors agi de la sorte à bon escient et 
volontairement dans la dissémination des drogues parmi les leurs, afin d'en tirer un profit pécuniaire, ou auraient-ils été pris à leur propre piège, débordés et dépassés par le phénomène ? Quoi qu'il en soit, les choses ne s'étant pas encore refroidies, et les événements de la guerre n'étant toujours pas dépassionnés, il est préférable d'attendre pour se prononcer avec plus de recul, et donc plus sûrement et plus librement sur cette question.

Cependant, l'observateur ne peut s'empêcher de se demander si cette négligence montrée par les responsables à l'égard de ce phénomène n'était pas due aussi à la constatation qu'ils faisaient que la consommation en groupe de la drogue renforçait le sentiment d'appartenance à l'ensemble, formant ainsi un élément de rapprochement à cause de ce besoin commun, de cette recherche collective, de ce plaisir partagé, de ces risques associés, de cette inconscience retrouvée chez tout un chacun. Ces états d'âmes renforçant le collectif n'auraient sans doute pas eu lieu sans le rattachement à la milice.

Celle-ci n'était d'ailleurs plus lieu de rattachement, mais lieu de dépendance. Et comme en Orient, le groupe a tendance à se confondre avec son leader et ses acolytes, la dépendance à la drogue se doublait d'une dépendance à la personne ; Hassan ben Sabbah renaissait-il de ses cendres en plusieurs "copies" ? La secte des Hashashins réapparaissait-elle non loin de ses lieux d'origine ? Pourquoi pas, même si cette "analyse", je le répète, n'est qu'une hypothèse parmi d'autres.

Quant à la guerre déclarée à la drogue par les responsables des milices, elle ne le fut qu'assez tard, quand tout le monde commençait à s'étonner de ce silence qui paraissait curieux pour certains et signe d'approbation pour les autres. Peuvent-ils dans ce cas arguer de la méconnaissance du problème ? Ce serait jeter le discrédit sur les services de renseignements dont chaque milice se flattait. Peuvent-ils parler de dramatisation et d'amplification du phénomène ? Les milliers de drogués et de drames humains sont là pour démentir cette assertion. Il fallait pourtant bien "réagir". Le choix a été porté sur une stratégie médiatique où la lutte contre la drogue eut lieu principalement contre quelques boucs émissaires ces derniers devant servir de preuves des changements d'attitudes.

12 C\&C : Y a-t-il eu une évolution du phénomène entre la période 75-77, celle des années 80 et celle des années 90 ?

13 A.B. : L'évolution du phénomène peut être divisée en deux périodes : la première s'étend aux deux premières années de la guerre en 1975-76. A cette époque, la propagation de la drogue eut lieu assez subrepticement parmi des jeunes qui n'y avaient guère touché auparavant, et qui vivaient dans un milieu social ne favorisant pas particulièrement la propagation de ce phénomène. Comme je l'ai signalé plus haut, ceux qui en usaient avant la guerre (c'est-à-dire une proportion minime au départ) ne cherchaient pas à s'afficher bien au contraire, ils le faisaient en cachette pour des raisons essentiellement sociales et parce que l'usage de la drogue était le plus souvent solitaire. D'autre part, abstraction faite de quelques petits trafiquants, de dealers, les responsables des milices étaient encore "novices" en la matière et ignorants quant à la portée du phénomène dont les dégâts étaient méconnus dans le pays. Je me souviens toujours des premiers entretiens que j'avais eu à ma demande au cours de la deuxième année de la guerre avec des responsables miliciens et politiques afin d'attirer leur attention sur la dangerosité de la prise de drogue par les combattants, et comment j'avais été écouté avec une oreille certes apparemment attentive mais avec un œil visiblement amusé, ce qui a fini à la longue par me frustrer et me décourager. 
La longue trêve d'une année (mi 77 - mi 78) que connut la guerre au Liban a contribué à mettre au grand jour ce phénomène de toxicomanie. Des spectacles jusqu'alors étrangers à la société libanaise apparaissaient dans les quartiers. Des groupes de jeunes, agressifs, violents; racoleurs se formaient au sein même du corps social et il s'agissait des enfants de nos voisins devenus méconnaissables! Certaines mères parlèrent de la "colère de Dieu", d'autres "d'un sort jeté", et les plus sensées, de mauvaises fréquentations au sein des milices où les petits trafiquants avaient toute impunité. Brusquement, dans les années 80 , une sorte de prise de conscience eut lieu chez quelques hauts dirigeants miliciens. Réaction qui se traduisit par une campagne de répression, physique au bas de la pyramide, et plus politico-policière au fur et à mesure que l'on grimpait dans la hiérarchie. Des "négociations" ne tardèrent pas à suivre. Elles aboutirent à un accord consistant à arrêter le trafic et la vente locale, à payer une certaine redevance pour "l'effort de guerre", en contrepartie de quoi la marchandise pouvait arriver tranquillement dans les différents ports, en traversant les "douanes" de chaque milice.

La deuxième période commence avec la reprise des combats qui ont alors duré de 1978 à 1990 avec des trêves insignifiantes. Le trafic local reprend alors de plus belle et le trafic extérieur s'organise sur une vaste échelle. Probablement enivrés par l'avant-goût des sommes mirobolantes rapportées par ce trafic, certains responsables s'y donnent à cœur joie et l'infrastructure humaine des réseaux s'étoffe et s'autonomise par milice (selon une intégration verticale). Entre temps, la consommation de drogues parmi les miliciens (toutes organisations confondues) bat son plein, la drogue se trouvant à profusion surtout après le foisonnement des laboratoires dits clandestins. Toutes les parties en présence sur le territoire libanais, toutes sans exception, y trouvent leur compte. C'était réellement la "poule aux œufs d'or"! A tel point que chacune des milices pouvait facilement se passer des subventions en armes et en argent des pays "protecteurs" et avait son propre budget qui se chiffrait en centaines de millions et pour certaines, en milliards de dollars. Parfois, il s'agissait aussi de troc d'armes versus des drogues. Des investissements économiques dans le pays et à l'étranger ont en outre servi à faire fructifier cet argent. Depuis octobre 90 la guerre semble être terminée. Les milices sont partiellement désarmées et politiquement plus ou moins contenues. Mais le potentiel économique accumulé n'est pas vraiment touché. Il le serait partiellement si disparaissait les redevances et les impôts qu'elles imposaient aux citoyens pour "effort de guerre" et en contrepartie de leur soi-disant "protection"... prélèvement qui sont vécus par l'immense majorité des gens comme une rançon, une extorsion de fonds, plutôt que comme une contribution. Seulement, la source principale de leurs revenus n'est pas encore touchée. On dirait qu'un modus vivendi est établi entre les parties en présence. Comment et pour quelles raisons ? Je pense qu'à partir de là, il serait plus prudent d'ignorer les faits et la suite des événements, surtout pour un citoyen qui n'est "protégé" que par sa science et sa profession médicale. 
INDEX

Mots-clés : entretien, drogue, guerre, contrebande et trafic

Index géographique : Liban 